\title{
Congenital Lobar Emphysema in Saudi Newborn
}

Abdulrahman Almesfer ${ }^{1}$, Abdullah Al Mutairi ${ }^{2}$, Mohammed Saleh Alfaleh ${ }^{3}$, Badi Al Enazi ${ }^{4 *}$

${ }^{1,2}$ Pediatric Resident at Alyammamh Hospital, Riyadh, Saudi Arabia

${ }^{3}$ Intern at king Khalid University Hospital Riyadh, Saudi Arabia

${ }^{4}$ Pediatric Consultant at Alyammamh Hospital, Riyadh, Saudi Arabia

DOI: $10.36347 /$ sjmcr.2020.v08i02.018

| Received: 06.02.2020 | Accepted: 14.02.2020 | Published: 16.02.2020

*Corresponding author: Dr. Badi Al Enazi

Abstract

Congenital lobar emphysema is a rare developmental anomaly of the lower respiratory tract that is characterized by hyperinflation of one or more of the pulmonary lobes. The diagnosis of Congenital Lobar Emphysema often can be diagnosed by chest radiograph and CT. Treatment of CLE with respiratory distress is the surgical resection of the affected lobe (Lobectomy). The prognosis is typically good to excellent. Here we present a case report for a newborn with cyanosis that starts immediately after birth.

Keywords: Congenital lobar emphysema, cyanosis.

Copyright @ 2020: This is an open-access article distributed under the terms of the Creative Commons Attribution license which permits unrestricted use, distribution, and reproduction in any medium for non-commercial use (NonCommercial, or CC-BY-NC) provided the original author and source are credited.

\section{INTRODUCTION}

Congenital lobar emphysema (CLE) is an overdistension of a pulmonary lobe leading to ventilation-perfusion mismatch [1]. It can be autosomal dominant or sporadic [2]. CLE is more common in males. It is more involved in the left lung lobe followed by the middle right lobe, and less in the upper right lobe and bilateral. Studies showed an association between CLE and congenital anomalies like double superior vena cava and horseshoe kidney [3].

Patients with CLE presented with respiratory signs and symptoms include tachypnea, dyspnea, wheezing, cough, decreased breath sounds and cyanosis, it is exaggerated by feeding or crying $[2,4,6]$.

CLE can be diagnosed by a chest radiograph. It may reveal hyperlucency on the affected side with ipsilateral atelectasis, widened spaces between the rib and flat diaphragm. The mediastinum may be displaced away from the affected side. CT scan can be more accurate [5]. Echocardiography should be performed to exclude atrial septal defect [2].

Resection of the affected lung parenchyma, it is the favorable method for the symptomatic patient. Emergency thoracotomy can be used in severe respiratory distress for life-saving [4, 6]. However, Long-term prognosis by surgery excision is good [2].

\section{Case Report}

A twenty-one days old Saudi boy, full-term, normal spontaneous vaginal delivery. He had a history of NICU admission for seven days due to cyanosis during feeding and a decrease in oxygen saturation. Since he was discharged from NICU according to the mother, he has persisting tachypnea, in the last two days, he started to have three attacks of cyanosis around his lips.

On physical examination, the patient looks ill, irritable, in respiratory distress and mildly dehydrated. His weight is $3.5 \mathrm{~kg}$ in 75 -percentile, height is $51 \mathrm{~cm} 50$ percentile Head circumference: $35 \mathrm{~cm} 50$-percentile. His tympanic temperature was $36.8^{\circ} \mathrm{c}$ and blood pressure was $98 / 44 \mathrm{mmHg}$. Pulse was regular with a rate of 160 beats per minute, respiratory rate was 68 per minute, and oxygen saturation is $88 \%$ on room air. Respiratory examination revealed decreased air entry in the left side. Cardiovascular examination revealed normal S1 and S2 with no murmur, and the central nervous system examination revealed no neurological deficits. Abdominal examination revealed soft and lax; there was no tenderness or organomegaly. The patient admitted to PICU, kept NPO, IV Fluid and antibiotics were started. Initially, the patient was on nasal CPAP but he doesn't improve and he intubated. Chest X-ray (Figure-1) showed hyperinflation in the left side with deviation of the mediastinum. CT scan correlates with a chest X-ray (Figure-2). A patient diagnosed as a case of Congenital lobar emphysema and Left thoracotomy and 


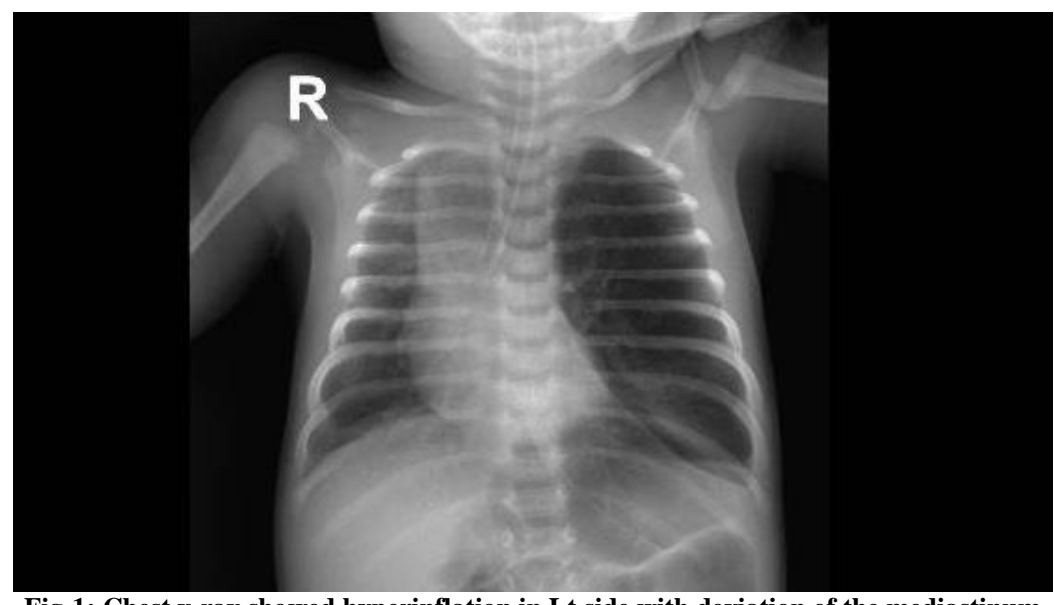

Fig-1: Chest x-ray showed hyperinflation in Lt side with deviation of the mediastinum



Fig-2: CT chest: showed Hyper inflate of Left upper lobe with mediastinal diffuse to the other side

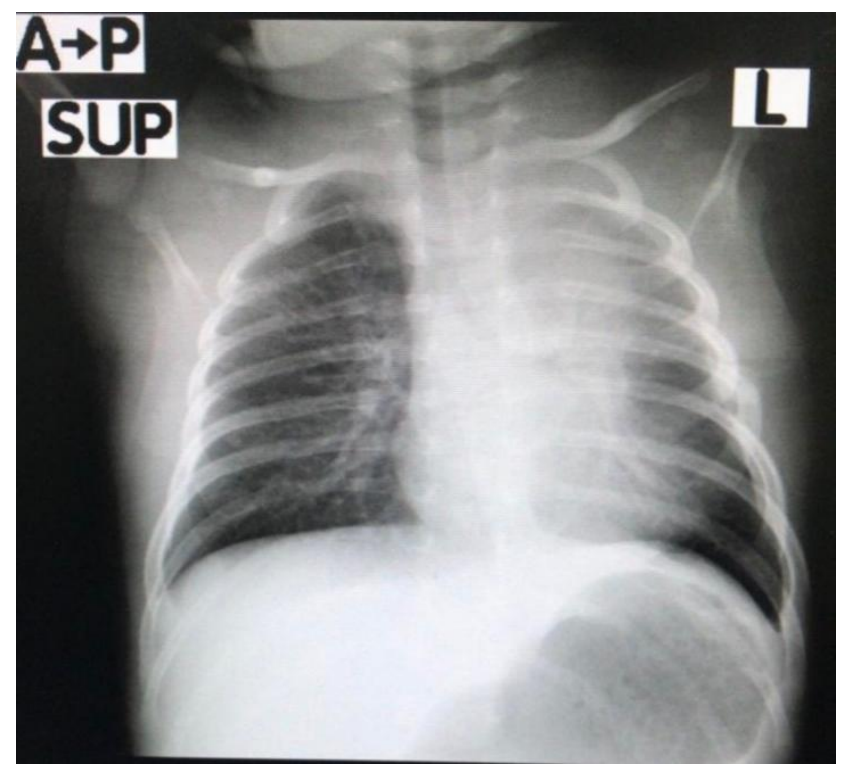

Fig-3: Post-operative chest $\mathrm{X}$ ray showed marked improvement 


\section{DiscuSSION}

In this case report, we present a 21-days male suffering CLE, the patent was suffering from cyanosis, tachypnea, respiratory distress, mild dehydration, and on chest examination he was found to have subcostal and substernal retraction and decrease air entry in the left side, the patient was diagnosed having CLE as his clinical presentations referred to it such as symptoms starts from few days to the first 6 month of life, tachypnea, cyanosis [7, 8]. Our patient was male and he was affected in his left side, it was reported that CLE is more common in males $[9,10]$ and the most affected lobe is the left upper lobe [7] as we found in our case. We also followed diagnosis strategy as it was reported that diagnosis is started with suspecting by clinical examination, then performing X-ray that can be confirmed by CT of the chest [11], X-ray was performed for our patient and the patient was kept NPO and started IV fluid. Although some authors reported that conservative treatment can be used for patients with mild symptoms [12-14], in a study of 15 years experience about the surgical management of CLE, it was reported that surgical management of CLE is the golden key for favorable and safe outcome [6]. In the present case, the patient was treated by surgical means where left thoracotomy and left lung upper lobectomy was done, the patient showed improvements and he was discharged. However, follow up should be performed for further cases for better prognosis and to manage any adverse outcomes.

\section{CONCLUSION}

Congenital lobar emphysema is a rare developmental anomaly of the lower respiratory tract that is characterized by hyperinflation of one or more of the pulmonary lobes. However, the left upper lobe is the most effective lobe. It is common in males more than females. CLE can be diagnosed by chest radiograph in the neonatal period or during early infancy. Treatment of CLE with respiratory distress is Lobectomy with a good prognosis.

DECLARATION OF PATIENT CONSENT: The authors certify that they have obtain all appropriate patient consent forms the family understand that the child name will not be published.

ACKNOWLEDGMENT: We greatly acknowledge to the patient family for their cooperation

FINANCIAL SUPPORT AND SPONSORSHIP: Nil.

Conflicts of Interest: There are no conflicts of interest.

\section{REFERENCES}

1. Walsh TA, Gopagondanahalli KR, Malhotra A. Williams-Beuren Syndrome and Congenital Lobar Emphysema: Uncommon Association with Common Pathology?. Case reports in pediatrics, 2017.

2. Latif I, Shamim, S, Ali S. Congenital lobar emphysema. Journal of Pakistan Medical Association, 2016, 66(2):210-212.

3. Badiu I, Hiriscau A, Lupan I, Samasca G. Congenital lobar emphysema in infants. Maedica, 2017; 12(2):133.

4. Gorostidi F, de Buys Roessingh A, Gonzalez M, Simon C, Monnier Y. Congenital lobar emphysema presenting as an airway foreign body. International journal of pediatric otorhinolaryngology, 2015;79(12):2450-2452.

5. Mulvany JJ, Weatherall A, Charlton A, Selvadurai H. Congenital lobar emphysema: diagnostic and therapeutic challenges. Case Reports. 2016 Jun 22;2016:bcr2016214994.

6. Mohamed EE, Ghaffar AM, Abdel-Aal KM, Helmy AA, Ashry M. Surgical management of congenital lobar emphysema: A 15 years experience in a tertiary thoracic surgery unit. Journal of the Egyptian Society of CardioThoracic Surgery. 2018 Dec 1;26(4):308-317.

7. Datta AK, Mandal S, Jana JK. Congenital lobar emphysema: a case report. Cases journal, 2009; 2(1):67.

8. Unnikrishnan KN. A Case Report On Congenital Lobar Emphysema. Asian Journal Pharm Clin Res, 2017; 10:5-6.

9. Cay A, Sarihan H. Congenital malformation of the lung. Journal Cardiovasc Surg (Torino); 2000; 41:507-510.

10. Al-Salem AH, Gyamfi YA, Grant CS. Congenital lobar emphysema. Can Journal Anaesth, 1990; 37:377-379.

11. Stanger P, Lucas Jr RV, Edwards JE. Anatomic factors causing respiratory distress in acyanotic congenital cardiac disease. Special reference to bronchial obstruction. Pediatrics; 1969; 43(5):760e9.

12. Ozcelik U, Gocmen A, Kiper N, Dogru D, Dilber E, Yalcin EG. Congenital lobar emphysema: evaluation and long-term follow-up of thirty cases at a single center. Pediatr Pulmonol; 2003; 35(5):384e91.

13. Roghair GD. Nonoperative management of lobar emphysema. Long-term follow-up. Radiology; 1972;102(1):125e7.

14. Shannon DC, Todres ID, Moylan FM. Infantile lobar hyperinflation: expectant treatment. Pediatrics; 1977; 59:1012e8. Suppl(6 Pt 2). 